\section{ORIGINAL RESEARCH}

\author{
Q. Yu \\ J. Yang \\ P. Wang
}

\title{
Malignant Tumors and Chronic Infections in the Masticator Space: Preliminary Assessment with In Vivo Single-Voxel ${ }^{1} \mathrm{H}-\mathrm{MR}$ Spectroscopy
}

\author{
BACKGROUND AND PURPOSE: Differential diagnosis between malignant tumors and chronic infections \\ in the masticator space remains challenging. The purpose of the study was to categorize various \\ changes of both lesions by using single-voxel ${ }^{1} \mathrm{H}-\mathrm{MR}$ spectroscopy.
}

\begin{abstract}
MATERIALS AND METHODS: Twenty-four masticator space lesions, 7 chronic infections, and 17 malignant tumors were assessed with ${ }^{1} \mathrm{H}-\mathrm{MR}$ spectroscopy before treatment procedures. The MR spectroscopic findings were compared with surgical and histopathologic results. Localization technique for ${ }^{1} \mathrm{H}-\mathrm{MR}$ spectroscopy was made by a point-resolved spectroscopy sequence at an echo time of $144 \mathrm{~ms}$. Choline (Cho) signals (identified at $3.2 \mathrm{ppm}$ ) and Cho signal intensity-to-noise ratio (Cho/noise) were considered as evaluating criteria.
\end{abstract}

\begin{abstract}
RESULTS: All the lesions were found with $3{ }^{1} \mathrm{H}-\mathrm{MR}$ spectroscopic types: type 1, lesions without Cho signals ( 3 chronic infections); type 2, lesions with Cho signals and Cho/noise ratio $<3$ (4 chronic infections and 4 malignant tumors); and type 3 , lesions with Cho signals and Cho/noise ratio $>3$ ( 13 malignant tumors). The mean $\pm \mathrm{SD}$ of the Cho/noise ratio between chronic infections and malignant tumors was $2.31 \pm 0.19$ and $5.76 \pm 3.29(P<.01)$, respectively.
\end{abstract}

CONCLUSION: In vivo single-voxel ${ }^{1} \mathrm{H}-\mathrm{MR}$ spectroscopy may be helpful in the assessment of masticator space lesions. Differences of Cho signals and Cho/noise ratios between malignant tumors and chronic infections provide valuable information in the differentiation of these 2 lesions.

D ifferentiation of chronic infections versus malignant tumors in the masticator space with CT and MR imaging is often difficult. To make an accurate diagnosis, one needs to explore new imaging techniques to analyze possible chemical or biochemical components of the lesions. Although MR spectroscopy was initially described with potential uses for head and neck lesions more than 20 years ago, ${ }^{1-4}$ it was not until recent years that the technology was widely used clinically. ${ }^{5}$ With rapid development and improvement of hardware and software, in vivo point-resolved MR spectroscopy $\left({ }^{1} \mathrm{H}-\mathrm{MR}\right.$ spectroscopy) has started to be used in the evaluation of extracranial head and neck lesions of the tongue, ${ }^{6-7}$ nasopharynx ${ }^{8}$ parotid gland, ${ }^{9}$ thyroid, ${ }^{10}$ and metastatic lymph nodes of the neck. ${ }^{6-8,10-12}$ In addition, the technology was used to detect recurrences of malignant tumors and to monitor treated or untreated tumors. ${ }^{5,11-13}$ However, to our knowledge, the ${ }^{1} \mathrm{H}-\mathrm{MR}$ spectroscopic appearance of chronic infections and malignant tumors in the masticator space has not been reported.

As one of the latest techniques for imaging analysis, singlevoxel ${ }^{1} \mathrm{H}$-MR spectroscopy measures living tissue metabolism at the molecular level. Increased choline (Cho) signals were generally thought to be an important criterion in assessing various tumors. However, whether the Cho metabolites could play a role in the differentiation of chronic infections and ma-

Received July 19, 2007; accepted after revision October 2

From the Department of Radiology (0.Y., P.W.), Ninth People's Hospital, School of Medicine, Shanghai Jiao Tong University, Shanghai, P. R. China; the Division of Oral and Maxillofacial Radiology (J.Y.), Temple University School of Dentistry, and the Department of Diagnostic Imaging, Temple University School of Medicine, Philadelphia, Pa.

Please address correspondence to Qiang Yu, DDS, Department of Radiology, Ninth People's Hospital, School of Medicine, Shanghai Jiao Tong University, Shanghai 200011, P. R. China; e-mail: yuqiang6155@163.com

DOI 10.3174/ajnr.A0897 lignant tumors in the masticator space was unknown. The aims of this preliminary study were to evaluate in vivo the single-voxel ${ }^{1} \mathrm{H}-\mathrm{MR}$ spectroscopic appearance of chronic infections and malignant tumors in the masticator space and to determine if differences exist between both lesions in terms of the levels of Cho metabolites.

\section{Materials and Methods}

\section{Subjects}

From 2003 to 2006, 47 patients with lesions in the masticator spaces were referred to the Department of Radiology. MR imaging and ${ }^{1} \mathrm{H}$-MR spectroscopy were performed for all patients. Three of the studies were unsuccessful because of uninterpretable spectra and unacceptable line width at half maximum of the unsuppressed water peak. Among the remaining 44 lesions, 24 were confirmed histopathologically to be either inflammatory lesions or malignant tumors. These 24 patients consisted of 18 men and 6 women, ranging in age from 3 to 82 years, with a mean age of 40.6 years. Pathologic outcomes of 24 lesions included 7 chronic infections, 4 squamous cell carcinomas, 7 salivary carcinomas, and 6 sarcomas. The demographic distribution of the subjects and lesions is listed in the Table.

\section{MR Imaging and MR Spectroscopy}

MR imaging and ${ }^{1} \mathrm{H}-\mathrm{MR}$ spectroscopy were performed on a $1.5 \mathrm{~T}$ Signa TwinSpeed system (GE Healthcare, Milwaukee Wis). A neurovascular array coil or a quadrature transmit/receive head coil was used. Axial MR imaging was performed by using T1-weighted spinecho and T2-weighted fast spin-echo sequences. Coronal MR imaging was performed by using a T2-weighted fast spin-echo sequence with fat suppression. The parameters of the T1-weighted spin-echo sequence were listed as follows: TR/TE, 600/11 ms; 3 acquisitions; FOV, $24 \mathrm{~cm}$; bandwidth, $31.25 \mathrm{~Hz}$; matrix, $256 \times 192$; section thickness, 5 $\mathrm{mm}$; spacing, $1 \mathrm{~mm}$. The parameters of the T2-weighted fast spin- 


\begin{tabular}{|c|c|c|c|c|c|}
\hline \multicolumn{6}{|c|}{ Single-voxel ${ }^{1} \mathrm{H}-\mathrm{MR}$ spectroscopic findings of chronic infections and malignant tumors in the masticator space } \\
\hline Case No./Sex/Age & Origin & Pathology & VOI $\left(\mathrm{cm}^{3}\right)$ & Cho Signal & Cho Signal-to-Noise Ratio \\
\hline $1 / F / 29$ & Unknown & Chronic infection & 8.00 & - & $\mathrm{N} / \mathrm{A}$ \\
\hline $2 / \mathrm{M} / 42$ & Unknown & Chronic infection & 8.39 & - & $\mathrm{N} / \mathrm{A}$ \\
\hline $3 / \mathrm{M} / 26$ & Odontogenic & Chronic infection & 11.73 & + & 2.50 \\
\hline 4/M/16 & Mandible & Chronic infection & 13.29 & - & N/A \\
\hline $5 / F / 71$ & Mandible & Chronic infection & 6.62 & + & 2.46 \\
\hline $6 / \mathrm{M} / 3$ & Odontogenic & Chronic infection & 10.48 & + & 2.13 \\
\hline $7 / F / 11$ & Mandible & Chronic infection & 5.52 & + & 2.15 \\
\hline $8 / \mathrm{M} / 49$ & Unknown & Adenoid cystic carcinoma & 8.00 & + & 6.49 \\
\hline $9 / F / 49$ & Maxillary sinus & Adenoid cystic carcinoma & 6.74 & + & 2.50 \\
\hline 10/M/39 & Maxillary sinus & Adenoid cystic carcinoma & 6.11 & + & 4.88 \\
\hline $11 / \mathrm{M} / 22$ & Unknown & Adenoid cystic carcinoma & 7.74 & + & 11.84 \\
\hline $12 / / \mathrm{M} / 70$ & Unknown & Adenocarcinoma & 12.48 & + & 3.47 \\
\hline 13/M/82 & Metastasis & Adenocarcinoma & 4.17 & + & 3.40 \\
\hline $14 / F / 49$ & Parotid gland & Mucoepithelial carcinoma & 10.11 & + & 7.20 \\
\hline 15/M/29 & Parotid gland & Rhabdomyosarcoma & 18.35 & + & 11.08 \\
\hline 16/M/33 & Unknown & Rhabdomyosarcoma & 5.12 & + & 4.95 \\
\hline $17 / M / 57$ & Unknown & Fibrosarcoma & 7.83 & + & 2.22 \\
\hline $18 / \mathrm{M} / 46$ & Unknown & Myxofibrosarcoma & 7.63 & + & 3.96 \\
\hline 19/M/32 & Mandible & Malignant fibrous histiocytoma & 7.99 & + & 2.30 \\
\hline $20 / \mathrm{M} / 20$ & Unknown & Synovial sarcoma & 7.76 & + & 10.25 \\
\hline $21 / F / 34$ & Unknown & Squamous cell carcinoma & 8.00 & + & 10.64 \\
\hline $22 / \mathrm{M} / 51$ & Oropharynx & Squamous cell carcinoma & 10.58 & + & 5.44 \\
\hline 23/M/49 & Maxillary sinus & Squamous cell carcinoma & 17.62 & + & 2.74 \\
\hline 24/M/70 & Soft palate & Squamous cell carcinoma & 7.88 & + & 4.56 \\
\hline
\end{tabular}

Note:-N/A indicates not available for measurement; VOI, volume of interest.

echo sequence were the following: axial TR/TE, 4700/85 ms and coronal TR/TE, 3200/100 ms; 3 acquisitions; echo-train length, 15; FOV, $24 \mathrm{~cm}$ (axial) and $20 \mathrm{~cm}$ (coronal); bandwidth, $19.23 \mathrm{~Hz}$ (axial) and $25 \mathrm{~Hz}$ (coronal); matrix, $256 \times 192$ (axial) and $320 \times 160$ (coronal); section thickness, $5 \mathrm{~mm}$; spacing, $1 \mathrm{~mm}$.

For lesion localization of ${ }^{1} \mathrm{H}-\mathrm{MR}$ spectroscopy, an axial MR imaging with a T2-weighted spin-echo sequence (TR/TE, 3780/80 ms) or T1 fluid-attenuated inversion recovery (TR/TE/TI, 2130/12/750 $\mathrm{ms}$ ) was performed with a matrix of $256 \times 160$, FOV of $24 \mathrm{~cm}$, and a section thickness of $5 \mathrm{~mm}$ with no intersection gap. A point-resolved spectroscopy sequence (PRESS, TR/TE/NEX, 1500/144/8; 64 signalintensity acquisitions) was used as the localizing technique. On the basis of the size of each lesion, the volume of interest (VOI) was adjusted to cover the depicted lesion area shown on the localizer images. The mean volume for all the lesions in this series was $9.09 \mathrm{~cm}^{3}$ with a range of $4.17-18.35 \mathrm{~cm}^{3}$.

The prescanning for ${ }^{1} \mathrm{H}-\mathrm{MR}$ spectroscopy automatically adjusted the received gains, the transmit gain, the center frequency, a 3-plane autoshim for prescribed VOI, and the water-suppression pulse. The full width at half maximum of the unsuppressed water peak in the PRESS sequence was $<17 \mathrm{~Hz}$. After the acquisition of MR spectra, all of the data were automatically processed by the PROBE/SV (Singlevoxel Proton Brain Examination, an image-guided clinical spectroscopy package provided by GE Healthcare, Milwaukee, Wis). The procedures of PROBE/SV include internal water-referenced phasecorrection, apodization with a gaussian filter (2.5- $\mathrm{Hz}$ width), 1-time zero-filling, fast Fourier transformation, baseline correction, registration of metabolic resonance peaks (Cho at $3.2 \mathrm{ppm}$ and creatine [Cr] at $3.0 \mathrm{ppm}$ ) and root-mean-square (RMS) noise, and integration of metabolite intensity based on the peak area. The RMS noise was measured by computing the value over two $300-\mathrm{Hz}$-wide regions of the spectrum (the range of frequencies $[-570.0 \mathrm{~Hz},-275.0 \mathrm{~Hz}]$ and $[365.0 \mathrm{~Hz}, 665.0 \mathrm{~Hz}])$ and taking the greater of the 2 levels. Subsequently, Cho signal intensity-to-noise ratio (Cho/noise) was mea- sured by a radiologist. The overall time of the examination was approximately 9 minutes, including neoplasm-localization sequences (4 minutes), prescanning (1 minute), and acquisition of PRESS sequence (TE of $144 \mathrm{~ms}$ ) and MR spectrum (4 minutes).

\section{Criteria and Statistical Analysis}

Cho signals and Cho/noise were regarded as the criteria in this study to determine if there were differences between chronic infections and malignant tumors. The Wilcoxon sign test, a nonparametric test, was used to compare differences of Cho/noise ratio between chronic infections and malignant tumors. A $P$ value $<.05$ was considered as a statistically significant difference.

\section{Results}

Of 24 masticator space lesions, 21 lesions (4 chronic infections and 17 malignant tumors) were identified with Cho signals (Figs 1 and 2), and the remaining 3 chronic infections had no Cho signals (Fig 3). Cho/noise ratios were measured in these 21 lesions. The detailed distribution of Cho signals and Cho/ noise ratio data for each lesion is listed in the Table. In addition, creatine (Cr) signals were found in 6 of 24 lesions (25\%), including 5 malignant tumors (Fig 2) and 1 chronic infection.

All the lesions could be classified into 3 types on the basis of Cho signals and Cho/noise ratios: type 1 (Fig 3), lesions with absence of Cho signals ( 3 chronic infections); type 2, lesions with Cho signals and a Cho/noise ratio $<3$ ( 4 chronic infections and 4 malignant tumors); and type 3 (Figs 1 and 2), lesions with Cho signals and a Cho/noise ratio $>3$ (13 malignant tumors). If type 3 lesions were considered as malignant tumors, the true-positive detection rate of malignant tumors was 13 of 17; the true-negative rate, 7 of 7 ; the false-positive lesion, none of 7; and the false-negative rate, 4 of 17 . Therefore, in vivo single-voxel ${ }^{1} \mathrm{H}-\mathrm{MR}$ spectroscopy had a sensitivity of $76.5 \%$, specificity of $100 \%$, positive predictive value of 

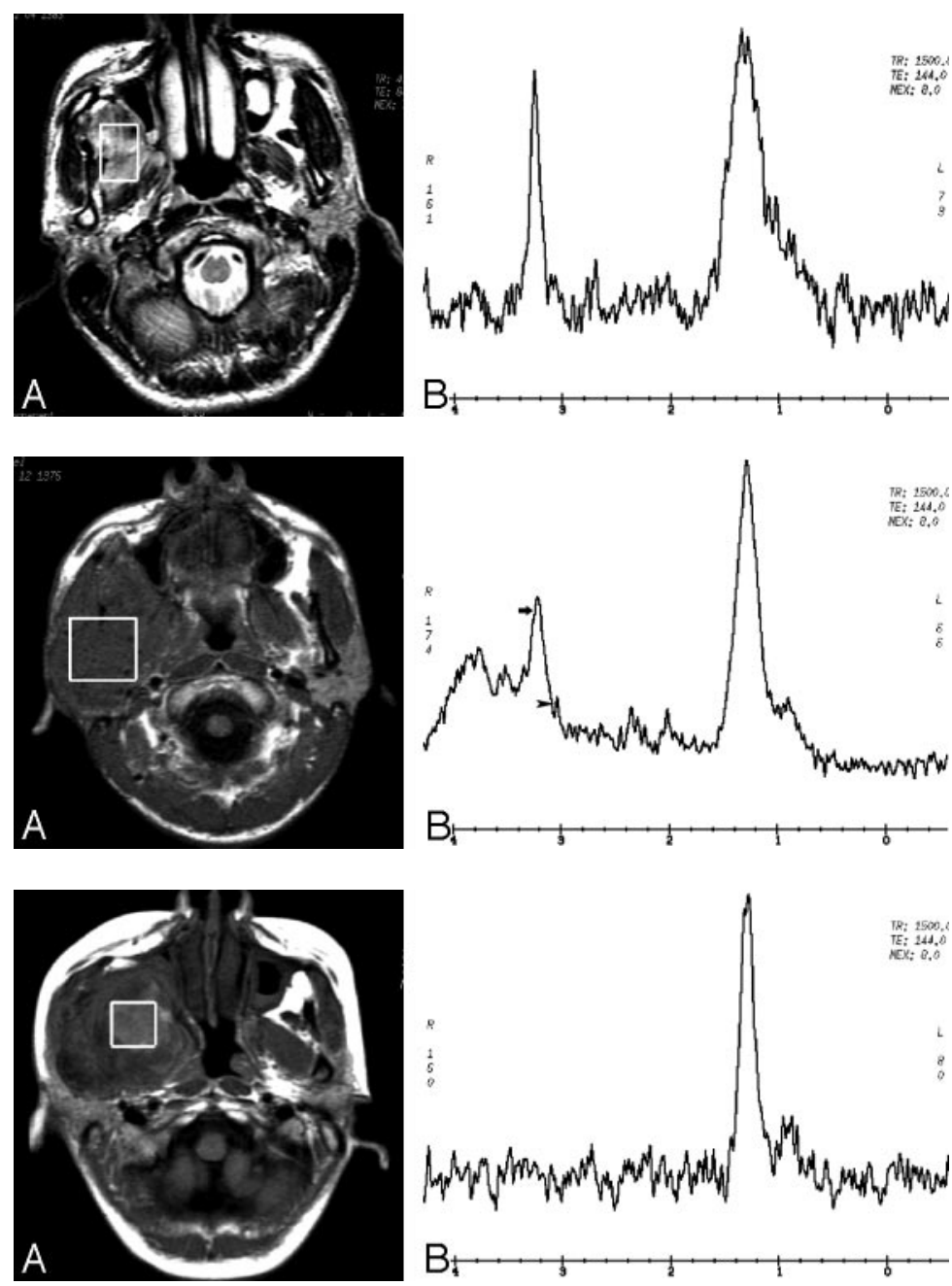

Fig 1. Case 11 with adenoid cystic carcinoma in the right masticator space. Axial T2-weighted image $(A)$ demonstrates that the lesion with high signa intensity is located in the right medial masticator space. The MR spectrum $(B)$ shows a distinct Cho peak at $3.2 \mathrm{ppm}$. The lipid signals are visible at $0.9-1.5 \mathrm{ppm}$.
Fig 2. Case 15 with rhabdomyosarcoma in the right parotid gland and masticator space. Axial T1-weighted image $(A)$ demonstrates the lesion with intermediate signal intensity in the right parotid gland and masticator space. On the MR spectrum (B), both Cho peak (arrow) and $\mathrm{Cr}$ peak (arrowhead) present at 3.2 and $3.0 \mathrm{ppm}$, respectively.
$100 \%$, negative predictive value of $63.6 \%$, and accuracy of $83.3 \%$.

The mean $\pm \mathrm{SD}$ of Cho/noise ratios for chronic infections and malignant tumors was $2.31 \pm 0.19$ and $5.76 \pm 3.29$, respectively. Statistically, there was a significant difference between both lesions $(P=.009)$.

\section{Discussion}

An early and correct differentiated diagnosis between an infection and a malignant tumor in the masticator space is pivotal for evaluating the prognosis of a patient. However, some chronic infections or inflammatory pseudotumors in the masticator spaces may be without any acute inflammatory signs, such as facial pain and swelling and pus formation, and are similar to the malignant tumors clinically. The inflammatory lesions in the extraorbital head and neck spaces can also mimic the malignant tumors on conventional CT and MR imaging. ${ }^{14-15}$ These lead to difficulty in indiscriminating between both kinds of lesions.

In vivo single-voxel ${ }^{1} \mathrm{H}-\mathrm{MR}$ spectroscopy is one of the latest techniques for analyzing molecular structures of living organisms. The height of Cho peaks shown on in vivo single-voxel ${ }^{1} \mathrm{H}$-MR spectroscopic images is believed to be related to increased cellular membrane phospholipid biosynthesis and is, therefore, considered as an active marker for cellular proliferation. ${ }^{16}$ A number of studies with in vivo ${ }^{1} \mathrm{H}-\mathrm{MR}$ spectroscopy have shown that Cho signals could be reliably found in most malignant tumors of the extracranial head and neck, such as squamous cell carcinomas, ${ }^{6-8,11-13,17}$ salivary carcinomas, ${ }^{9,12}$ and extracranial malignant lymphomas. ${ }^{11,18}$ Soft-tissue and bone sarcomas (outside the head and neck) marked with Cho signals have also been reported. ${ }^{19}$ However, there were few studies describing the in vivo ${ }^{1} \mathrm{H}-\mathrm{MR}$ spectroscopic appearances of inflammatory lesions. Shah et $\mathrm{al}^{5}$ reported that ${ }^{1} \mathrm{H}$-MR spectroscopy could be used to differentiate recurrent head and neck tumors from posttreatment changes. The inflammatory responses with formation of granulation tissue in the tumor bed might result from radiation therapy. The reactive changes after radiation therapy had a low Cho/Cr ratio or absence of Cho and its derivatives. ${ }^{5}$ The current study also showed that the chronic infections in the masticator space had either absence of Cho signals or low Cho/noise ratios. On the contrary, all malignant lesions in the space presented with Cho signals and most of them with high Cho/noise ratios. The different MR spectroscopic appearances between the chronic infections and malignant tumors might be due to the fact that there were hypervascularity, hypercellularity, and higher levels of Cho metabolites and its derivatives.

In the current study, Cho/noise ratio was used to evaluate masticator space lesions. Previous reports ${ }^{20,21}$ indicated that if the Cho/noise ratio was $\geq 2$, the lesion was most likely a malignant tumor. The data of this study demonstrated similar 
results in the masticator space. The chronic infections were either without Cho signals or had a Cho/noise ratio $<3$, whereas most of malignant tumors had a Cho signal intensity and a Cho/noise ratio $>3$. Also, there was a statistically significant difference between the mean Cho/noise ratios of chronic inflammatory lesions and malignant tumors. On the basis of these differences, in vivo single-voxel ${ }^{1} \mathrm{H}-\mathrm{MR}$ spectroscopy might be useful in differentiating chronic infections from malignant tumors in the masticator space.

On the basis of the literature review, the most common semiquantitative criterion used for evaluation of extracranial head and neck neoplasms with ${ }^{1} \mathrm{H}-\mathrm{MR}$ spectroscopy was the Cho/Cr ratio. ${ }^{6-13,17}$ However, some studies found that the Cho/Cr ratio could only be measured in half or less of extracranial head and neck neoplasms. ${ }^{8-11}$ In our series, the peaks of $\mathrm{Cr}$ metabolites could only be identified in approximately $25 \%$ of masticator space lesions. This might be due to the fact that $\mathrm{Cr}$ peaks were at $3.0 \mathrm{ppm}$ and could not be separated from the background noise. The difficulty in detecting $\mathrm{Cr}$ signals made the criterion of Cho/Cr ratio less useful in the assessment of masticator space lesions. The Cho/noise ratio has previously been applied to evaluate malignant tumors elsewhere in the body. ${ }^{20,21}$ Furthermore, we found that it was easier to measure the Cho/noise ratio than the $\mathrm{Cho} / \mathrm{Cr}$ ratio in the masticator space lesions. The RMS noise can be automatically measured by the MR imaging scanner with the same algorithm and method during each acquisition of the MR spectrum.

The drawbacks with in vivo single-voxel ${ }^{1} \mathrm{H}-\mathrm{MR}$ spectroscopy in masticator space lesions should be pointed out. First, shimming difficulty existed in this study due to considerable magnetic susceptibility differences in the extracranial head and neck. ${ }^{6,11}$ Unavoidable inclusion of adjacent bony and aircontaining structures in the large VOI reduced the likelihood of obtaining an adequate shim, which resulted in decreased spectral resolution. ${ }^{6}$ Second, there was a $23.5 \%$ (4/17) falsenegative rate for malignant tumors in the current study. This indicated that there was overlap of the MR spectroscopic findings between chronic infections and malignant tumors with the current standards. Third, the VOI used in the current study was $>4 \mathrm{~cm}^{3}$, which limited its use for small lesions. Using a VOI $<4 \mathrm{~cm}^{3}$ in the masticator space may result in failure of in vivo single-voxel ${ }^{1} \mathrm{H}$-MR spectroscopy. Therefore, other physiologic techniques, such as MR diffusion-weighted imaging and dynamic enhanced MR imaging, might have potential in the assessment of small masticator space lesions. Finally, because the current study was preliminary and the sample size for both lesions was relatively small, further studies with larger sample sizes are required to validate these findings.

\section{Conclusion}

The current study demonstrated that there were 3 different types of $1 \mathrm{H}-\mathrm{MR}$ spectroscopic manifestations in both masticator space lesions. The differences of Cho signals and Cho/ noise ratios among these 3 manifestations might be potentially useful in the differentiation of chronic infections from malignant tumors in the masticator space.

\section{References}

1. Mafee MF, Barany M, Gotsis ED, et al. Potential use of in vivo proton spectroscopy for head and neck lesions. Radiol Clin North Am 1989;27:243-54

2. Dawson DE, Sekhar V, Pearson G, et al. Nuclear magnetic resonance spectroscopy of plasma for the detection of head and neck cancer. Am J Otolaryngol 1989;10:244-49

3. Scher RL, Ropka ME, Neal DA, et al. NMR spectroscopy evaluation of plasma "oncolipids" in head and neck cancer. Otolaryngol Head Neck Surg 1990;102:34-40

4. Chow JM, Gotsis ED, Jarc M, et al. Proton nuclear magnetic resonance spectroscopy of plasma lipoproteins in head and neck cancer patients. Am J Otolaryngol 1990;11:332-38

5. Shah GV, Gandhi D, Mukherji SK. Magnetic resonance spectroscopy of head and neck neoplasms. Top Magn Reson Imaging 2004;15:87-94

6. Mukherji SK, Schiro S, Castillo M, et al. Proton MR spectroscopy of squamous cell carcinoma of the extracranial head and neck: in vitro and in vivo studies. AJNR Am J Neuroradiol 1997;18:1057-72

7. Star-Lack JM, Adalsteinsson E, Adam MF, et al. In vivo 1H-MR spectroscopy of human head and neck lymph node metastasis and comparison with oxygen tension measurements. AJNR Am J Neuroradiol 2000;21:183-93

8. King AD, Yeung DKW, Ahuja AT, et al. In vivo proton MR spectroscopy of primary and nodal nasopharyngeal carcinoma. AJNR Am J Neuroradiol 2004;25:484-90

9. King AD, Yeung DKW, Ahuja AT, et al. Salivary gland tumors at in vivo proton MR spectroscopy. Radiology 2005;237:563-69

10. King AD, Yeung DKW, Ahuja AT, et al. In vivo $1 \mathrm{H}$ MR spectroscopy of thyroid carcinoma. Eur J Radiol 2004;54:112-17

11. King AD, Yeung DKW, Ahuja AT, et al. Human cervical lymphadenopathy: evaluation with in vivo 1H-MRS at 1.5 T. Clinical Radiol 2005;60:592-98

12. Bisdas $S$, Baghi $M$, Huebner $F$, et al. In vivo proton $M R$ spectroscopy of primary tumours, nodal and recurrent disease of the extracranial head and neck. Eur Radiol 2007;17:251-57

13. Bezabeh T, Odlum $\mathrm{O}$, Nason R, et al. Prediction of treatment response in head and neck cancer by magnetic resonance spectroscopy. AJNR Am J Neuroradiol 2005;26:2108-13

14. Som PM, Curtin HD. Parapharyngeal and masticator space lesions. In: Som PM, Curtin HD, eds. Head and Neck Imaging. St Louis: Mosby; 2003:1996

15. Vuysere SD, Hermans R, Sciot R, et al. Extraorbital inflammatory pseudotumor of the head and neck: CT and MR findings in three patients. AJNR Am J Neuroradiol 1999;20:1133-39

16. Rulz-Cabello J, Cohen JS. Phospholipid metabolites as indicators of cancer cell function. NMR Biomed 1992;5:226-33

17. Maheshwari SR, Mukherji SK, Neelon B, et al. The choline/creatine ratio in five benign neoplasms: comparison with squamous cell carcinoma by use of in vitro MR spectroscopy. AJNR Am J Neuroradiol 2000;21:1930-35

18. Schwarz AJ, Maisey NR, Collins DJ, et al. Early in vivo detection of metabolic response: a pilot study of $1 \mathrm{H}$ MR spectroscopy in extrcranial lymphoma and germ cell tumors. Br J Radiol 2002;75:956-66

19. Wang CK, Li CW, Hsieh TJ, et al. Characterization of bone and soft-tissue tumors with in vivo ${ }^{1} \mathbf{H}$ MR spectroscopy: initial results. Radiology 2004;232:599-605

20. Bartella L, Morris EA, Dershaw DD, et al. Proton MR spectroscopy with choline peak as malignant marker improves positive predictive value for breast cancer diagnosis: preliminary study. Radiology 2006;239:686-92. Epub 2006 Apr 7

21. Lee J, Yamaguchi T, Abe A, et al. Clinical evaluation of choline measurement by proton MR spectroscopy in patients with malignant tumors. Radiat Med 2004;22:148-54 